CREAT. MATH. INFORM.

Volume 29 (2020), No. 2,

Pages $113-120$

\title{
Ball convergence theorem for inexact Newton methods in Banach space
}

\author{
IOANNIS K. ARgYros and SANTHOSH GEORGE
}

\begin{abstract}
We present a local convergence analysis for inexact Newton methods in order to approximate a solution of a nonlinear equation in a Banach space. Our sufficient convergence conditions involve only hypotheses on the first Fréchet-derivative of the operator involved. Numerical examples are also provided in this study.
\end{abstract}

\section{INTRODUCTION}

Many problems in computational sciences and other disciplines can be brought in the form of the nonlinear equation

$$
F(x)=0,
$$

where $F$ is a Fréchet-differentiable operator defined on a subset $D$ of a Banach space $X$ with values in a Banach space $Y$. In this study we are concerned with the problem of approximating a solution $x^{*}$ of equation (1.1). In Numerical Functional Analysis, for finding solution of (1.1) is essentially connected to Newton-like methods [1]- [27]. The Newton-like methods are usually studied based on: semi-local and local convergence. The semi-local convergence matter is, based on the information around an initial point, to give conditions ensuring the convergence of the iterative procedure; while the local one is, based on the information around a solution, to find estimates of the radii of convergence balls. There exist many studies which deal with the local and semi-local convergence analysis of Newton-like methods such as [1]- [27].

We present a local convergence analysis for inexact Newton method defined for each $n=0,1,2, \cdots$ by

$$
\begin{aligned}
y_{n} & =x_{n}-\alpha F^{\prime}\left(x_{n}\right)^{-1} F\left(x_{n}\right), \\
x_{n+1} & =x_{n}-z_{n}
\end{aligned}
$$

where $x_{0}$ is an initial point, $\alpha$ is a parameter and $\left\{z_{n}\right\} \subset X$ is a given sequence. Many iterative methods are special cases of method (1.2).

Newton's method [3-7,26,27]: Choose $\alpha=1$ and $z_{n}=x_{n}-y_{n}$, then, we get

$$
x_{n+1}=x_{n}-F^{\prime}\left(x_{n}\right)^{-1} F\left(x_{n}\right) .
$$

Two-step Newton's method I [3-7, 26, 27]: Choose $\alpha=1$ and $z_{n}=F^{\prime}\left(x_{n}\right)^{-1} F\left(y_{n}\right)$, then, we get

$$
\begin{aligned}
y_{n} & =x_{n}-F^{\prime}\left(x_{n}\right)^{-1} F\left(x_{n}\right), \\
x_{n+1} & =x_{n}-F^{\prime}\left(x_{n}\right)^{-1} F\left(y_{n}\right) .
\end{aligned}
$$

Received: 30.12.2019. In revised form: 25.08.2020. Accepted: 31.08.2020 2010 Mathematics Subject Classification. 65D10, 65D99.

Key words and phrases. High convergence order method, Banach space, Local Convergence, Fréchet-derivative..

Corresponding author: Santhosh George; sgeorge@nitk.edu.in 
Two-step Newton's method II [3-7,26,27]: Choose $\alpha=1$ and $z_{n}=x_{n}-y_{n}+F^{\prime}\left(x_{n}\right)^{-1} F\left(y_{n}\right)$, then, we get

$$
\begin{aligned}
y_{n} & =x_{n}-F^{\prime}\left(x_{n}\right)^{-1} F\left(x_{n}\right), \\
x_{n+1} & =y_{n}-F^{\prime}\left(y_{n}\right)^{-1} F\left(y_{n}\right) .
\end{aligned}
$$

Weerakoon-Fernando method I [24]: Choose $\alpha=1$ and $z_{n}=\left[I+A_{n}^{-1} B_{n}\right] F^{\prime}\left(x_{n}\right)^{-1} F\left(x_{n}\right)$, then, we get

$$
\begin{aligned}
y_{n} & =x_{n}-F^{\prime}\left(x_{n}\right)^{-1} F\left(x_{n}\right), \\
x_{n+1} & =x_{n}-\left[I+A_{n}^{-1} B_{n}\right] F^{\prime}\left(x_{n}\right)^{-1} F\left(x_{n}\right) .
\end{aligned}
$$

where $A_{n}=F^{\prime}\left(x_{n}\right)+F^{\prime}\left(y_{n}\right)$ and $B_{n}=F^{\prime}\left(x_{n}\right)-F^{\prime}\left(y_{n}\right)$.

King and Ostrowski method [27]: For $X=Y=S, S=\mathbb{R}$ or $S=\mathbb{C}$

$$
\alpha=\frac{2}{3}, z_{n}=\frac{1}{2}\left(1+\frac{1}{1+\frac{3}{2}\left(\frac{F^{\prime}\left(y_{n}\right)}{F^{\prime}\left(x_{n}\right)}-1\right)}\right) F^{\prime}\left(x_{n}\right)^{1} F\left(x_{n}\right)
$$

or

$$
\alpha=1, z_{n}=x_{n}-y_{n}-\frac{F\left(y_{n}\right)}{F^{\prime}\left(x_{n}\right)} \frac{F\left(x_{n}\right)}{F\left(x_{n}\right)-2 F\left(y_{n}\right)} .
$$

Amat et all method (scalar case) [3]: Choose $z_{n}=x_{n}-y_{n}+\alpha F^{\prime}\left(x_{n}\right)^{-1} F\left(y_{n}\right)$ to obtain

$$
\begin{aligned}
y_{n} & =x_{n}-\alpha F^{\prime}\left(x_{n}\right)^{-1} F\left(x_{n}\right), \\
x_{n+1} & =y_{n}-\alpha F^{\prime}\left(x_{n}\right)^{-1} F\left(y_{n}\right) .
\end{aligned}
$$

The last two methods require the existence of the fourth derivative. Many other choices for $\alpha$ or $z_{n}$ are possible [1]- [27]. In general the convergence of these methods requires the existence of second, fourth or even higher order derivatives although only the first derivative appears in these methods [11,12,20,21,25].

Moreover, in the proof Taylor series expansions are used for methods (1.5)-(1.9) or other specializations of method (1.2) requiring the existence of such high order derivatives.

These assumptions however are very restrictive. As a motivational example, let us define function $f$ on $D=\left[-\frac{1}{2}, \frac{5}{2}\right]$ by

$$
f(x)=\left\{\begin{array}{l}
x^{3} \ln x^{2}+x^{5}-x^{4}, x \neq 0 \\
0, \quad x=0
\end{array}\right.
$$

Choose $x^{*}=1$. We have that

$$
\begin{aligned}
f^{\prime}(x) & =3 x^{2} \ln x^{2}+5 x^{4}-4 x^{3}+2 x^{2}, f^{\prime}(1)=3, \\
f^{\prime \prime}(x) & =6 x \ln x^{2}+20 x^{3}-12 x^{2}+10 x \\
f^{\prime \prime \prime}(x) & =6 \ln x^{2}+60 x^{2}-24 x+22 .
\end{aligned}
$$

Then, obviously, function $f^{\prime \prime \prime}$ is unbounded on $D$. Hence, the results e.g. in method, (1.5)(1.9) cannot be used. In the present paper we only use hypotheses on the first Fréchet derivative (see conditions (2.16)-(2.19)). This way, we expand the applicability of method (1.2).

Our idea so far has been used on methods (1.3)-(1.9) and some others [1-8] (with the exception of (2.10) and (2.21) not needed for special cases). But it is obvious from the above that a unified approach for studing these methods is needed. Here is where the motivation and the novelty of our paper lies, since method (1.2) generalizes so many methods. Moreover, we have not done this before at this generalization.

The rest of the paper is organized as follows. The local convergence of method (1.2) is given in Section 2, whereas the numerical examples are given in Section 3. Some comments are given in the concluding section 4 . 


\section{LOCAL CONVERGENCE ANALYSIS}

We present the local convergence analysis of method (1.2) in this section. Let $U(v, \rho), \bar{U}(v, \rho)$ stand, respectively, for the open and closed balls in $X$ with center $v \in X$ and radius $\rho>0$. Let $L_{0}>0, L>0, M>0, p \in[0,1]$ and $\alpha \in S(S=\mathbb{R}$ or $S=\mathbb{C})$ be given parameters. Suppose that there exists a continuous nondecreasing function $\psi:\left[0, \frac{1}{L_{0}}\right) \rightarrow[0,+\infty)$ such that

$$
\psi(t) \rightarrow+\infty \text { or } \psi(t) \rightarrow a \geq 0 \text { as } t \rightarrow{\frac{1}{L_{0}}}^{-} .
$$

It is convenient for the local convergence analysis of method (1.2) that follows to define functions $g_{1}, g_{2}$ and $h_{2}$ on the interval $\left[0, \frac{1}{L_{0}}\right)$ by

$$
\begin{aligned}
& g_{1}(t)=\frac{1}{2\left(1-L_{0} t\right)}[L t+2 M|1-\alpha|], \\
& g_{2}(t)= \begin{cases}\frac{L t}{2\left(1-L_{0} t\right)}+\psi(t), & p=0 \\
\frac{L t}{2\left(1-L_{0} t\right)}+\psi(t) t^{p}, & p \neq 0\end{cases}
\end{aligned}
$$

and

$$
h_{2}(t)=g_{2}(t)-1
$$

Moreover, define parameters

$$
r_{1}=\frac{2(1-M|1-\alpha|)}{2 L_{0}+L}
$$

and

$$
r_{A}=\frac{2}{2 L_{0}+L}
$$

Suppose that

$$
M|1-\alpha|<1 .
$$

Then, we have that $g_{1}\left(r_{1}\right)=1$,

and

$$
0<r_{1}<r_{A}<\frac{1}{L_{0}}
$$

$$
0 \leq g_{1}(t)<1 \text { for each } t \in\left[0, r_{1}\right) .
$$

We also get that

Suppose that

$$
h_{2}(0)= \begin{cases}\psi(0)-1, & p=0 \\ -1, & p \neq 0\end{cases}
$$

$$
\psi(0)<1 \text {, if } p=0 .
$$

Then $h_{2}(0)<0$ for each $p \in[0,1]$ and $h_{2}\left(r_{A}\right)=\left\{\begin{array}{ll}\psi\left(r_{A}\right)>0, & p=0 \\ \psi\left(r_{A}\right) r_{A}^{p}>0, & p \neq 0\end{array}\right.$. If follows from the intermediate value theorem that function $h_{2}$ has zeros in the interval $\left(0, r_{A}\right)$. Denote by $r_{2}$ the smallest such zero

$$
r=\min \left\{r_{1}, r_{2}\right\}<r_{A} .
$$

Then, we have that for each $t \in[0, r)$

$$
\begin{aligned}
& 0 \leq g_{1}(t)<1 \\
& 0 \leq g_{2}(t)<1 .
\end{aligned}
$$

Next, we present the local convergence analysis of method (1.2) using the preceding notation. 
Theorem 2.1. Let $F: D \subseteq X \rightarrow Y$ be a Fréchet-differentiable operator. Suppose that there exist $x^{*} \in D$, parameters $L_{0}>0, L>0, M>0$ and $\alpha \in S$ and a function $\psi$ satisfying (2.10), (2.12) (if $p=0),(2.11)$

$$
\begin{gathered}
F\left(x^{*}\right)=0, F^{\prime}\left(x^{*}\right)^{-1} \in L(Y, X), \\
\left\|F^{\prime}\left(x^{*}\right)^{-1}\left(F^{\prime}(x)-F^{\prime}\left(x^{*}\right)\right)\right\| \leq L_{0}\left\|x-x^{*}\right\|, \text { for each } x \in D \\
\left\|F^{\prime}\left(x^{*}\right)^{-1}\left(F^{\prime}(x)-F^{\prime}(y)\right)\right\| \leq L\|x-y\|, \text { for each } x, y \in D_{0}=D \cap U\left(x^{*}, \frac{1}{L_{0}}\right) \\
\left\|F^{\prime}\left(x^{*}\right)^{-1} F^{\prime}(x)\right\| \leq M \text { for each } x \in D_{0} \\
\bar{U}\left(x^{*}, r\right) \subseteq D_{0},
\end{gathered}
$$

and there exists $z: D \subseteq X \rightarrow X$ such that for each $x \in D_{0}, F^{\prime}(x)^{-1} \in L(Y, X)$ and for some $p \in[0,1]$

$$
\left\|F^{\prime}(x)^{-1} F(x)-z(x)\right\| \leq \psi\left(\left\|x-x^{*}\right\|\right)\left\|x-x^{*}\right\|^{1+p} .
$$

Then, the sequence $\left\{x_{n}\right\}$ generated by method (1.2) for $x_{0} \in U\left(x^{*}, r_{2}\right)$ is well defined, remains in $U\left(x^{*}, r\right)$ for each $n=0,1,2, \cdots$ and converges to $x^{*}$. Moreover, the following estimates hold

$$
\left\|y_{n}-x^{*}\right\| \leq g_{1}\left(\left\|x_{n}-x^{*}\right\|\right)\left\|x_{n}-x^{*}\right\|<\left\|x_{n}-x^{*}\right\|<r,
$$

and

$$
\left\|x_{n+1}-x^{*}\right\| \leq g_{2}\left(\left\|x_{n}-x^{*}\right\|\right)\left\|x_{n}-x^{*}\right\|<\left\|x_{n}-x^{*}\right\|,
$$

where the " $g$ " functions are defined previously. Furthermore, for $T \in\left[r, \frac{2}{L_{0}}\right)$ the limit point $x^{*}$ is the only solution of equation $F(x)=0$ in $D_{1}=D \cap \bar{U}\left(x^{*}, T\right)$.

Proof. We shall show estimates (2.22) and (2.23) using mathematical induction. By hypothesis $x_{0} \in U\left(x^{*}, r\right)$, the definition of $r$ and (2.17), we get that

$$
\left\|F^{\prime}\left(x^{*}\right)^{-1}\left(F^{\prime}\left(x_{0}\right)-F^{\prime}\left(x^{*}\right)\right)\right\| \leq L_{0}\left\|x_{0}-x^{*}\right\|<L_{0} r<1 .
$$

Then, by the the Banach Lemma on invertible operators [4,5], we get that

$$
\left\|F^{\prime}\left(x_{0}\right)^{-1} F^{\prime}\left(x^{*}\right)\right\| \leq \frac{1}{1-L_{0}\left\|x_{0}-x^{*}\right\|} .
$$

Using the first substep in method (1.2) for $n=0,(2.14),(2.18),(2.19)$ and (2.25), we get in turn that

$$
\begin{aligned}
\left\|y_{0}-x^{*}\right\| \leq & \left\|x_{0}-x^{*}-F^{\prime}\left(x_{0}\right)^{-1} F^{\prime}\left(x_{0}\right)\right\|+|1-\alpha|\left\|F^{\prime}\left(x_{0}\right)^{-1} F\left(x^{*}\right)\right\| \\
& \times\left\|\int_{0}^{1} F^{\prime}\left(x^{*}\right)^{-1} F^{\prime}\left(x^{*}+\theta\left(x_{0}-x^{*}\right)\right)\left(x_{0}-x^{*}\right) d \theta\right\| \\
\leq & \left\|F^{\prime}\left(x_{0}\right)^{-1} F^{\prime}\left(x_{0}\right)\right\|\left\|\int_{0}^{1} F^{\prime}\left(x^{*}\right)^{-1}\left[F^{\prime}\left(x^{*}+\theta\left(x_{0}-x^{*}\right)\right)-F^{\prime}\left(x_{0}\right)\right]\left(x_{0}-x^{*}\right) d \theta\right\| \\
\leq & \frac{L\left\|x_{0}-x^{*}\right\|^{2}}{2\left(1-L_{0}\left\|x_{0}-x^{*}\right\|\right)}+\frac{|1-\alpha| M \mid\left\|x_{0}-x^{*}\right\|}{1-L_{0}\left\|x_{0}-x^{*}\right\|} \\
= & g_{1}\left(\left\|x_{0}-x^{*}\right\|\right)\left\|x_{0}-x^{*}\right\| \\
< & \left\|x_{0}-x^{*}\right\|<r,
\end{aligned}
$$


which shows (2.22) for $n=0$ and $y_{0} \in U\left(x^{*}, r\right)$. Then, using the second substep of method (1.2) for $n=0,(2.15),(2.21)$ and (2.26) we get for $z\left(x_{0}\right)=z_{0}$ that

$$
\begin{aligned}
\left\|x_{1}-x^{*}\right\| & \leq\left\|x_{0}-x^{*}-F^{\prime}\left(x_{0}\right)^{-1} F\left(x_{0}\right)\right\|+\left\|F^{\prime}\left(x_{0}\right)^{-1} F\left(x_{0}\right)-z_{0}\right\| \\
& \leq \frac{L\left\|x_{0}-x^{*}\right\|^{2}}{2\left(1-L_{0}\left\|x_{0}-x^{*}\right\|\right)}+\psi\left(\left\|x_{0}-x^{*}\right\|\right)\left\|x_{0}-x^{*}\right\|^{1+p} \\
& =g_{2}\left(\left\|x_{0}-x^{*}\right\|\right)\left\|x_{0}-x^{*}\right\|<\left\|x_{0}-x^{*}\right\|<r,
\end{aligned}
$$

which shows (2.23) and $x_{1} \in U\left(x^{*}, r\right)$. By simply replacing $x_{0}, y_{0}, z_{0}, x_{1}$ by $x_{k}, y_{k}, z_{k}, x_{k+1}$ in the preceding estimates we arrive at estimate (2.22) and (2.23). It then follows from the estimate $\left\|x_{k+1}-x^{*}\right\| \leq c\left\|x_{k}-x^{*}\right\|<r, c=g_{2}\left(\left\|x_{0}-x^{*}\right\|\right) \in[0,1)$ that $x_{k+1} \in U\left(x^{*}, r\right)$ and $\lim _{k \rightarrow \infty} x_{k}=x^{*}$.

Finally, to show the uniqueness part, let $y^{*} \in D_{1}$ be such that $F\left(y^{*}\right)=0$. Set $Q=$ $\int_{0}^{1} F^{\prime}\left(y^{*}+t\left(x^{*}-y^{*}\right) d t\right.$. Then using (2.17), we get that

$$
\begin{aligned}
\left\|F^{\prime}\left(x^{*}\right)^{-1}\left(Q-F^{\prime}\left(x^{*}\right)\right)\right\| & \leq \int_{0}^{1} L_{0}\left\|y^{*}+t\left(x^{*}-y^{*}\right)-x^{*}\right\| d t \\
& \leq \int_{0}^{1}(1-t)\left\|x^{*}-y^{*}\right\| d t \leq \frac{L_{0}}{2} T<1,
\end{aligned}
$$

it follows from (2.27) that $Q^{-1} \in L(Y, X)$. Then, from the identity $0=F\left(x^{*}\right)-F\left(y^{*}\right)=$ $Q\left(x^{*}-y^{*}\right)$, we deduce that $x^{*}=y^{*}$.

Remark 2.1. 1. In view of (2.17) and the estimate

$$
\begin{aligned}
\left\|F^{\prime}\left(x^{*}\right)^{-1} F^{\prime}(x)\right\| & =\left\|F^{\prime}\left(x^{*}\right)^{-1}\left(F^{\prime}(x)-F^{\prime}\left(x^{*}\right)\right)+I\right\| \\
& \leq 1+\left\|F^{\prime}\left(x^{*}\right)^{-1}\left(F^{\prime}(x)-F^{\prime}\left(x^{*}\right)\right)\right\| \leq 1+L_{0}\left\|x-x^{*}\right\|
\end{aligned}
$$

condition (2.19) can be dropped and $M$ can be replaced by

$$
M(t)=1+L_{0} t .
$$

2. The results obtained here can be used for operators $F$ satisfying autonomous differential equations [4] of the form

$$
F^{\prime}(x)=P(F(x))
$$

where $P$ is a continuous operator. Then, since $F^{\prime}\left(x^{*}\right)=P\left(F\left(x^{*}\right)\right)=P(0)$, we can apply the results without actually knowing $x^{*}$. For example, let $F(x)=e^{x}-1$. Then, we can choose: $P(x)=x+1$.

3. Suppose that there exists $L_{1}>0$ such that for each $x, y \in D$

$$
\left\|F^{\prime}\left(x^{*}\right)^{-1}\left(F^{\prime}(x)-F^{\prime}(y)\right)\right\| \leq L_{1}\|x-y\| .
$$

Set $\bar{r}_{A}=\frac{2}{2 L_{0}+L_{1}}$. Then, we have

$$
L_{0} \leq L_{1} \text {, and } L \leq L_{1}
$$

so $\bar{r}_{A} \leq r_{A}$ and if $L<L_{1}$, then $\bar{r}_{A}<r_{A}$. The radius $\bar{r}_{A}$ was shown by us to be the convergence radius of Newton's method [2,4,5]

$$
x_{n+1}=x_{n}-F^{\prime}\left(x_{n}\right)^{-1} F\left(x_{n}\right) \text { for each } n=0,1,2, \cdots
$$

under the conditions (2.17) and (2.18). It follows from the definition of $r$ (see 2.13)) that the convergence radius $r$ of the method (1.2) cannot be larger than the convergence radius $\bar{r}_{A}$ (or $r_{A}$ ) of the second order Newton's method (2.30). As already noted in [4,5] $\bar{r}_{A}$ is at least as large as the convergence ball given by Rheinboldt [26]

$$
r_{R}=\frac{2}{3 L_{1}} \text {. }
$$


In particular, for $L_{0}<L_{1}$ we have that

$$
r_{R}<\bar{r}_{A}
$$

and

$$
\frac{r_{R}}{\bar{r}_{A}} \rightarrow \frac{1}{3} \text { as } \frac{L_{0}}{L_{1}} \rightarrow 0 .
$$

That is our convergence ball $\bar{r}_{A}$ is at most three times larger than Rheinboldt's. The same value for $r_{R}$ was given by Traub [27].

4. It is worth noticing that method (1.2) is not changing when we use the conditions of Theorem 2.1 instead of the stronger conditions used in earlier studies. Moreover, we can compute the computational order of convergence (COC) defined by

$$
\xi=\ln \left(\frac{\left\|x_{n+1}-x^{*}\right\|}{\left\|x_{n}-x^{*}\right\|}\right) / \ln \left(\frac{\left\|x_{n}-x^{*}\right\|}{\left\|x_{n-1}-x^{*}\right\|}\right)
$$

or the approximate computational order of convergence

$$
\xi_{1}=\ln \left(\frac{\left\|x_{n+1}-x_{n}\right\|}{\left\|x_{n}-x_{n-1}\right\|}\right) / \ln \left(\frac{\left\|x_{n}-x_{n-1}\right\|}{\left\|x_{n-1}-x_{n-2}\right\|}\right) .
$$

This way we obtain in practice the order of convergence in a way that avoids the bounds involving estimates using estimates higher than the first Fréchet derivative of operator $F$.

5. Let us choose $z_{n}=F^{\prime}\left(x_{n}\right)^{-1} F\left(y_{n}\right)$ for each $n=0,1,2, \cdots$ and

$$
\psi(t)=\frac{|\alpha| M^{2}}{\left(1-L_{0} t\right)^{2}}
$$

for each $t \in\left[0, r_{A}\right]$. Then, condition (2.21) is satisfied for $p=0$. Indeed, we have that

$$
\begin{aligned}
\left\|F^{\prime}\left(x_{n}\right)^{-1} F\left(x_{n}\right)-z_{n}\right\| & =\left\|F^{\prime}\left(x_{n}\right)^{-1} F\left(x_{n}\right)-F^{\prime}\left(x_{n}\right)^{-1} F\left(y_{n}\right)\right\| \\
& \leq\left\|F^{\prime}\left(x_{n}\right)^{-1} F\left(x^{*}\right)\right\|\left\|\int_{0}^{1} F^{\prime}\left(x^{*}\right)^{-1} F^{\prime}\left(y_{n}+\theta\left(x_{n}-y_{n}\right)\right)\left(x_{n}-\right) d \theta\right\| \\
& \leq \frac{M\left\|y_{n}-x_{n}\right\|}{1-L_{0}\left\|x_{n}-x^{*}\right\|} \\
& \leq \frac{M|\alpha|\left\|F^{\prime}\left(x_{n}\right)^{-1} F^{\prime}\left(x^{*}\right)\right\|\left\|F^{\prime}\left(x^{*}\right)^{-1} F\left(x_{n}\right)\right\|}{1-L_{0}\left\|x_{n}-x^{*}\right\|} \\
& \leq \frac{M^{2}|\alpha|\left\|x_{n}-x^{*}\right\|}{\left(1-L_{0}\left\|x_{n}-x^{*}\right\|\right)^{2}}=\psi\left(\left\|x_{n}-x^{*}\right\|\right)\left\|x_{n}-x^{*}\right\| .
\end{aligned}
$$

Then, condition (2.12) becomes

$$
|\alpha| M^{2}<1
$$

and we can set

$$
a=\frac{|\alpha| M^{2}}{\left(1-L_{0} r_{A}\right)^{2}},
$$

so that (2.10) is also satisfied. 


\section{NumERICAL EXAMPLES}

We present numerical examples in this section in the case when $\psi(t)$ is given by (2.32).

Example 3.1. Let $X=Y=\mathbb{R}^{3}, D=\bar{U}(0,1)$. Define $F$ on $D$ for $v=(x, y, z)^{T}$ by

$$
\left.F(v)=\left(e^{x}-1, \frac{e^{x}-1}{2} y^{2}+y, z\right)\right)^{T} .
$$

Then, the Fréchet derivative is given by

$$
F^{\prime}(v)=\left[\begin{array}{ccc}
e^{x} & 0 & 0 \\
0 & (e-1) y+1 & 0 \\
0 & 0 & 1
\end{array}\right]
$$

Notice that $x^{*}=(0,0,0)^{T}, F^{\prime}\left(x^{*}\right)=F^{\prime}\left(x^{*}\right)^{-1}=\operatorname{diag}\{1,1,1\}$, so $L_{0}=e-1<L_{1}=$ $e, L=M=e^{\frac{1}{L_{0}}}$. Then, we have for $\alpha=0.7206$,

$$
\bar{r}_{A}=0.3249, r_{A}=0.3827, r_{1}=0.1913=r, r_{2}=0.2230 .
$$

Example 3.2. Returning back to the motivational example at the introduction of this study, we have $L_{0}=L_{1}=L=96.6629073, M=1.0631$. Then we have for $\alpha=0.5297$,

$$
\bar{r}_{A}=r_{A}=0.0069, r_{1}=0.0034=r, r_{2}=0069 .
$$

Example 3.3. Let $X=Y=C[0,1]$, the space of continuous functions defined on $[0,1]$ and be equipped with the max norm. Let $D=\bar{U}(0,1)$. Define function $F$ on $D$ by

$$
F(\psi)(x)=\psi(x)-5 \int_{0}^{1} x \theta \psi(\theta)^{3} d \theta
$$

We have that

$$
F^{\prime}(\psi(\xi))(x)=\xi(x)-15 \int_{0}^{1} x \theta \psi(\theta)^{2} \xi(\theta) d \theta, \text { for each } \xi \in D .
$$

Then, we get that $x^{*}=0, L_{0}=7.5, L=L_{1}=15=M, \alpha=0.9333$ and

$$
\bar{r}_{A}=r_{A}=0.0889, r_{1}=0.0444, r_{2}=0.0232=r .
$$

\section{CONCLUSION}

We present a local convergence analysis of inexact Newton method in order to approximate a solution of an equation in a Banach space setting. Earlier convergence analysis is based on hypotheses on higher than the first Fréchet-derivative. In this paper the local convergence analysis is based only on Lipschitz hypotheses of the first Fréchet-derivative. Hence, the applicability of these methods is expanded.

\section{REFERENCES}

[1] Amat, S., Busquier, S. and Gutierrez, J. M., Geometric construction of iterative functions to solve nonlinear equations, J. Comput. Appl. Math., 157 (2003), 197-205

[2] Amat, S., Hernández, M. A. and Romero, N., A modified Chebyshev's iterative method with at least sixth order of convergence, Appl. Math. Comput., 206 (2008), No. 1, 164-174

[3] Amat, S. Busquier, S. and Magreñań, Á. A., Reducing chaos and bifurication in Newton-ttype methods, Abstract and Applied Analysis, Vol. (2013), Article ID:726701

[4] Argyros, I. K., "Convergence and Application of Newton-type Iterations", Springer, 2008

[5] Argyros, I. K. and Hilout, S., Computational methods in nonlinear Analysis, World Scientific Publ. Co. , New Jersey, USA, 2013

[6] Argyros, I. K. and George, S., Mathematical modeling for the solution of equations and systems of equations with applications, Volume-III, Nova Publishes, NY, 2019 
[7] Argyros, I. K. and Magreñán, A. A., Iterative method and their dynamics with applications, CRC Press, New York, USA, 2017

[8] Argyros, I. K. and Magreñán, A. A., A contemporary study of iterative methods, Elsevier (Academic Press), New York, 2018.

[9] Arroyo, V., Cordero, A. and Torregrosa, J. R., Approximation of artificial intelligence satellites preliminary orbits: The efficiency challenge, Math. Comput. Modelling, 54 (2011), 1802-1807

[10] Candela, V. and Marquina, A.,Recurrence relations for rational cubic methods I: The Halley method, Computing, 44 (1990), 169-184

[11] Cordero, A. and Torregrosa, J., Variants of Newton's method using fifth order quadrature formulas, Appl. Math. Comput., 190 (2007), 686-698

[12] Chun, C., Some improvements of Jarratt's method with sixth-order convergence, Appl. Math. Comput., 190 (1990), No. 2, 1432-1437

[13] Ezquerro, J. A. and Hernández, M. A., A uniparametric Halley-type iteration with free second derivative, Int. J. Pure and Appl. Math., 6 (2003), No. 1, 99-110

[14] Ezquerro, J. A. and Hernández, M. A., New iterations of R-order four with reduced computational cost, BIT Numer. Math., 49 (2009), 325-342

[15] Ezquerro, J. A. and Hernández, M. A., On the R-order of the Halley method, J. Math. Anal. Appl., 303 (2005), 591-601

[16] Gutiérrez, J. M. and Hernández, M. A., Recurrence relations for the super-Halley method, Computers Math. Appl., 36 (1998), No. 7, 1-8

[17] Ganesh, M. and Joshi, M. C., Numerical solvability of Hammerstein integral equations of mixed type, IMA J. Numer. Anal., 11 (1991), 21-31

[18] Hernández, M. A., Chebyshev's approximation algorithms and applications, Computers Math. Appl., 41 (2001), No. (3-4), 433-455

[19] Hernández, M. A. and Salanova, M. A., Sufficient conditions for semi-local convergence of a fourth order multipoint iterative method for solving equations in Banach spaces, Southwest J. Pure Appl. Math., 1 (1999), 29-40

[20] Jarratt, P., Some fourth order multipoint methods for solving equations, Math. Comput., 20 (1966), No. 95, 434-437

[21] Kou, J. and Li, Y., An improvement of the Jarratt method, Appl. Math. Comput., 189 (2007), 1816-1821

[22] Parhi, S. K. and Gupta, D. K., Semi-local convergence of a Stirling-like method in Banach spaces, Int. J. Comput. Methods, 7 (2010), No. 02, 215-228

[23] Parhi, S. K. and Gupta, D. K., Recurrence relations for a Newton-like method in Banach spaces, J. Comput. Appl. Math., 206 (2007), No. 2, 873-887

[24] Petkovic, M. S., Neta, B. Petkovic, L. and Dzunic, J., Multi-point methods for solving nonlinear equations, Elsevier, 2013

[25] Ren, H., Wu, Q. and Bi, W., New variants of Jarratt method with sixth-order convergence, Numer. Algorithms, 52 (2009), No. 4, 585-603

[26] Rheinboldt, W. C., An adaptive continuation process for solving systems of nonlinear equations, In: Mathematical models and numerical methods (A. N. Tikhonov et al. eds.) pub. 3, (19), 129-142 Banach Center, Warsaw Poland

[27] Traub, J. F., Iterative methods for the solution of equations, Prentice Hall Englewood Cliffs, New Jersey, USA, 1964

\section{DEPARTMENT OF MATHEMATICAl SCIENCES}

CAMERON UNIVERSITY

LAWTON, OK 73505, USA

Email address: iargyrosecameron.edu

Department of MATHEMATICAl ANd Computational SCIENCES

NATIONAL INSTITUTE OF TECHNOLOGY KARNATAKA

SURATHKAL-575025, INDIA

Email address: sgeorge@nitk.edu. in 